\title{
Management and outcomes of an acute mid substance medial patella femoral ligament repairs in adolescents
}

\begin{abstract}
Background: Traumatic patellar dislocation with mid substance medial patellofemoral ligament (MPFL) tear is addressed with a MPFL repair. There is no definitive protocol detailing postoperative management of MPFL repairs. Using an open MPFL repair followed by physical therapy, we created a standardized protocol to optimize outcomes and return patients to previous levels of activity.
\end{abstract}

Methods: We retrospectively reviewed the charts of 23 patient athletes, aged 11 to 18 years (mean, 15.2years), who experienced a traumatic patellar dislocation with subsequent MPFL injury and intra-articular pathology who were treated according to our protocol between 2006 and 2009. We analyzed the clinical results of this cohort at each of their followups with interval radiographic imaging. Patients were evaluated based on time until return to play and recurrence of patellar dislocation or instability. On final assessment, patients completed the Kujala knee score.

Results: Mean time to return to play was 7 months. Of the patients who underwent surgical intervention and our perioperative rehabilitation protocol, three had recurrent dislocations. Patients with clinical and radiographic documentation of recovery achieved nearly $100 \%$ of their baseline function and reported that they were able to function at pre injury levels. 8 patients completed the Kujala knee score (average score, 96.3). Recovery times and return to play did not vary based on mechanism of injury, sex, or sport activity. Conclusion: Acceptable results can be obtained following traumatic patellar dislocation and subsequent surgical mid substance MPFL repair in adolescents using a defined surgical and rehabilitative algorithm.

Keywords: future complications, patellofemoral instability, osteochondral defects
Volume 3 Issue I - 2015

\author{
Nirav H Amin,' Shannon Safier, ${ }^{2}$ Ben Parker, ${ }^{2}$ \\ Neil Kumar, ${ }^{2}$ Daniel Hass, ${ }^{3}$ Martin Herman ${ }^{2}$ \\ 'Department of Orthopedic Surgery, Loma Linda University, \\ USA \\ ${ }^{2}$ Department of Orthopaedic Surgery, Drexel University \\ College of Medicine, USA \\ ${ }^{3}$ Department of Orthopaedic Surgery Cleveland, The Cleveland \\ Clinic Foundation, USA
}

Correspondence: Nirav Amin, Associate Professor, Orthopedic Surgery, Loma Linda University, California, USA, Email naminmd@gmail.com

Received: March 30, 2015 | Published: August 12, 2015
Abbreviations: MPFL, medial patella femoral ligament; MRI, magnetic resonance imaging; MVIC, maximal voluntary contraction

\section{Introduction}

Traumatic patellar dislocations remain a common acute knee disorder in adolescents. ${ }^{1}$ While debate continues regarding the advantages of surgical versus conservative treatment, adolescents with a primary patellar dislocation and articular injury are at greater risk for re-injury and loss of function. ${ }^{2,3}$ Thus, intervention and rehabilitation must be centered on preventing future complications, assessing risk for re-injury and restoring function. Fisher B et al. ${ }^{4}$ performed a systemic review of medial patella femoral ligament (MPFL) injuries reported between 1950 and 2010 and noted a need for a more comprehensive protocol for functional rehabilitation and return to sports. An algorithm that standardizes postoperative rehabilitation may aid in improved clinical outcomes and restoration of optimal function.

Although the MPFL is the primary restraint to patellofemoral instability, other biomechanical and anatomical factors, such as passive lateral hyper mobility of the patella, dysplasia of the lateral femoral trochlea, atrophy of the vastus medial is obliquus muscle, a high or lateral position of the patella and increased Q-angle have all been identified as possible predictors of redislocation. ${ }^{1,3}$ Osteochondral defects identified on imaging have also been used as a predictor for outcome. Osteochondral lesions associated with MPFL ruptures have been shown to cause increased pain, ${ }^{5}$ and may warrant alteration of a standard MPFL treatment protocol.

We have devised a standardized management protocol for mid substance MPFL disruption following traumatic patellar dislocation with concomitant chondral injury in adolescents. To our knowledge, this is the first study documenting a management algorithm for such injuries and may allow for efficient management and timely return to previous levels of activity.

\section{Materials and methods}

We retrospectively reviewed the charts of 23 patients with acute patellar dislocations treated between December 2006 and December 2009. Inclusion was based on three criteria: first-time acute patellar dislocation, no history of knee surgery and magnetic resonance imaging (MRI) findings consistent with a mid-substance MPFL tear with intra-articular pathology including chondral injury. All patients were treated with an open MPFL repair, arthroscopic management of intra-articular pathology addressing the patella lesion, protected weight bearing, then aggressive postoperative physical therapy. Patients with an isolated MPFL tear (i.e., without intra-articular pathology, no radiographic imaging of loose bodies and/or clinical symptoms), MRI findings consistent with an MPFL lesion along the femur or tibia, or history of confounding conditions were excluded. Three patients were eliminated from the study owing to their history of nail-patellar syndrome, Ehlers-Danlos syndrome and multiple 
recurrent dislocations, respectively. One patient was lost to follow-up, leaving a cohort of 19 patients.

All patients underwent an initial diagnostic MRI after a confirmed lesion on X-ray with either a loose body and/or fragment noted from the initial X-ray. Based on the presence or absence of a chondral defect, the patient underwent either surgical or nonsurgical management with physical therapy. The non-operative arm of this cohort was not the subject of this study. Patients were then followed for up to 84 months (45 to 84 months), to record Kujala scores postoperatively. The Drexel University College of Medicine institutional review board approved the study.

\section{Imaging evaluation}

A standardized protocol was used during the initial evaluation of all patients starting with their first office visit. For each patient who presented with symptoms consistent with patellar subluxation and mechanical intra-articular pathology, a standard MRI without contrast enhancement of the injured knee was obtained. We assume that in a patient who has recently sustained a patellar dislocation, MRI will show attenuation and/or rupture of the MPFL. Balcarek P et al. ${ }^{6}$ determined the importance of a preoperative MRI in ensuring adequate fixation with an MPFL repair. Therefore, the purpose of the MRI is to evaluate for the presence of concomitant injuries that could cause continued instability or pain. Seeley $\mathrm{M}$ et al. ${ }^{7}$ noted that adolescents with MPFL pathology have multiple sites of MPFL pathology and that more than $33 \%$ have intra-articular pathology. In all cases showing chondral lesions on MRI, surgical intervention was performed, consisting of arthroscopy to address intra-articular pathology, followed by an open MPFL repair. The MRI findings were confirmed by the senior orthopedic surgeon and by a fellowshiptrained musculoskeletal radiologist.

\section{Surgical technique}

Diagnostic arthroscopy is used to define all intra-articular pathology. Soft tissue injuries including partial meniscal tears, synovitis and chondromalacia are addressed first, followed by osteochondral defects along the patella and/or lateral femoral condyle. Depending on the extent of the chondral injury and the quality and size of the detached fragment (Figure 1), either internal fixation of the fragment or a microdrilling technique is employed. If internal fixation was employed, often the pathology was addressed after the arthroscopic procedure was completed. The medial border of the patella is evaluated arthroscopically. Finally, a medial parapatellar incision measuring 3 to $4 \mathrm{~cm}$ is made. Dissection is carried through the skin, subcutaneous tissue and musculo-ligamentous structures until the MPFL remnant is identified. The ligament is assessed at both the patellar and femoral insertions. In the cases reviewed here, the remnant was large enough to be repaired using six to eight 2-0 poly (ethylene, terephthalate) sutures, using a pants-over-vest technique with the knee in $30^{\circ}$ of flexion (Figure 2). The tears were found to be midsubstance and not avulsed from either the patella or femoral insertion. A varus stress is then applied to the patella to confirm adequate tensioning of the repair before the knee is taken through the full range of motion to assess patellar tracking. A standard closure of the subcutaneous tissues (Figure 3) is performed with 2-0 Vicryl (polyglactin 910; Ethicon, Somerville, NJ) before the skin is closed with a running Monocryl stitch (poliglecaprone 25; Ethicon). At the conclusion of the surgery, the knee is placed in an immobilizer, which

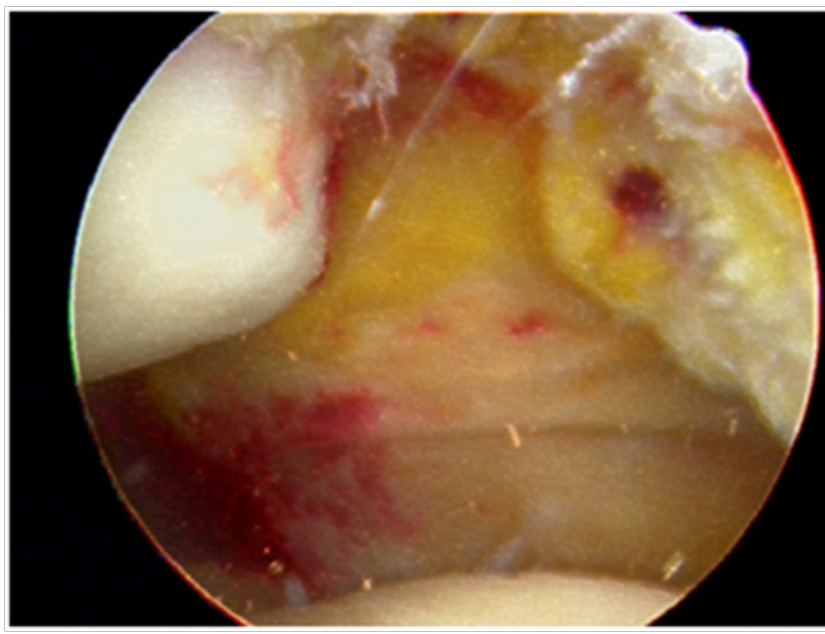

Figure I Arthroscopic visualization of patellar injury.

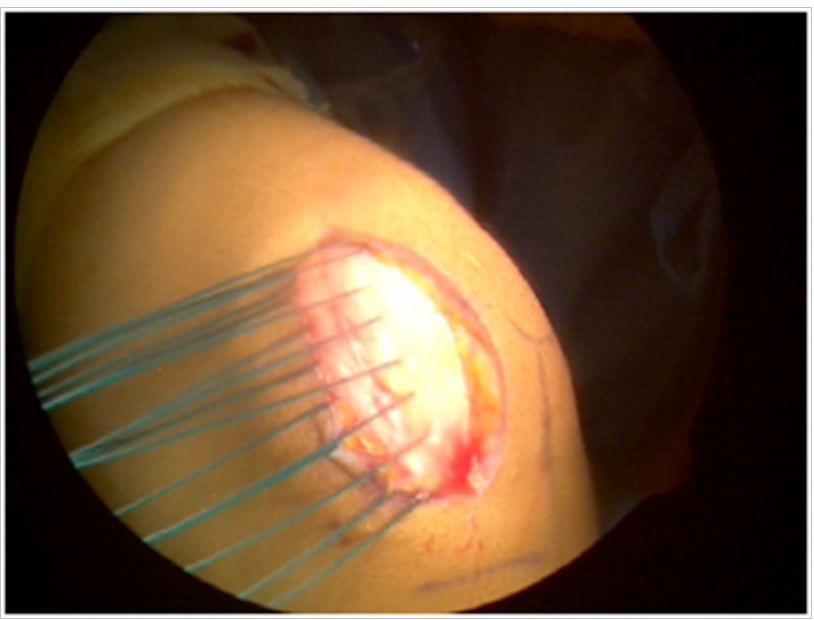

Figure 2 Medial patellofemoral ligament repair using suture.

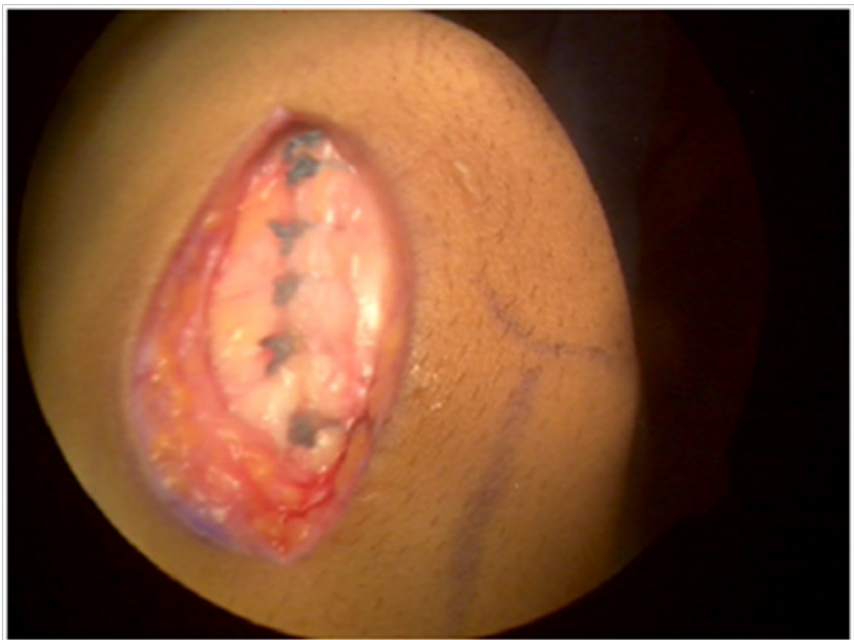

Figure 3 Closure of the medial patellofemoral ligament repair. the patient must wear until the first postoperative visit. 


\section{Post-operative rehabilitation}

Our standardized postoperative protocol begins with strict non weight bearing in a knee immobilizer due to the intra-articular pathology addressed in concurrence with the MPFL repair. The patient follows up within 1 week to evaluate the incision. During the first visit, the patient is transitioned into a Bledsoe brace (Bledsoe, Grand Prairie, TX) and allowed to start passive and active-assist range of motion from $0^{\circ}$ to $60^{\circ}$ under the guidance of a physical therapist. The patient must continue to avoid weight bearing for the first 4 weeks postoperatively to protect the repair and to avoid lateral patellar displacement, most prominent at $12^{\circ}$ to $30^{\circ}$ of flexion. ${ }^{8}$ Once the 4-week period of non weight bearing is completed, the patient is progressed to full weight bearing as tolerated. At this point, the Bledsoe brace is discontinued and physical therapy interventions are progressed to address impairments and functional limitations as outlined below.

Of particular importance is neuromuscular re-education of the quadriceps, as this muscle group has shown considerable weakness and activation deficits following knee surgery. ${ }^{9}$ In addition, good quadriceps control is required for normal gait and optimal patellofemoral arthrokinematics. Initially, the patient lies supine with a towel roll under the popliteal fossa and the physical therapist uses verbal and tactile cues to encourage volitional contraction of the quadriceps as the patient gently pushes down into towel. The patient is instructed to avoid proximal compensations and to focus on a quality contraction of the quadriceps. This is a slow and controlled movement pattern and the patient is instructed not to maximally tighten the muscle. The physical therapist may use tactile cues on the distal quadriceps to aid in the neuromuscular re-education of this muscle group. Once a voluntary quadriceps contraction is assessed to be adequate by the physical therapist, active assisted straight leg raises are commenced. This assistance is provided by either the physical therapist assisting to raise the leg, or by instructing the patient in the use of a long towel wrapped around the foot to allow the upper extremities to assist with the movement. The patient is cued to contract the quadriceps, then slowly lift the leg up 12 to 18 inches, with careful concentration to maintain the knee in a locked out extension position. This exercise is progressed with decreased levels of assistance until the patient can perform 20 straight leg raises without assistance to 18 inches with no quadriceps lag. Ideally, this goal should be obtained by 3 weeks following surgery while the patient is non-weight bearing. From this point, the patient is progressed to weight bearing beginning with simple weight shifts with upper extremity support, allowing the knee to flex and extend with partial weight through the surgical limb to promote functional quadriceps control. This is also done in a forward to backward manner to promote weight shifting in a functional walking position. Gait training with a focus on a heel-toe pattern, as well as appropriate knee flexion/extension and lumbopelvic control is the top priority once weight-bearing restrictions have been lifted at 4 weeks.

Just as with quadriceps exercises, initial interventions and exercises for the hip musculature focus on proper recruitment and progress from less challenging to more challenging. ${ }^{9}$ This may start very basically with gluteal isometrics in supine where the patient is cues to "squeeze your buttock muscles together" which has shown to recruit the gluteus maximus to $81 \%$ of maximal voluntary contraction (MVIC).$^{10}$ The side-lying clam shell demonstrates 53\% MVIC gluteus maximus and $47 \%$ MVIC gluteus medius and is performed in side lying with the knees partially flexed (Figure 4). The patient keeps the feet together and gently lifts the top knee up toward the ceiling, squeezing the gluteal muscles. Once adequate control has been established with these two exercises, side-lying hip abduction (51\% MVIC gluteus maximus and 63\% MVIC gluteus medius) is introduced (Figure 5). The therapist ensures that the patient is not compensating with his/her hip flexors and that the hip is in slight extension and external rotation to ensure the gluteals are being targeted. ${ }^{10-12}$ The Physical Therapist may need to use verbal and tactile cueing for gluteal contraction, as an important criteria to progress is the ability to voluntarily contract the gluteals. The patient must have an awareness of basic activation patterns before progressing to more functional positions. Although these non-weight bearing positions are excellent for teaching voluntary activation patterns, the patient needs to be progressed to weight bearing exercises to utilize these muscles in a more functional manner. The body weight squat is used as a starting point to teach appropriate lower extremity mechanics that can be carried over to multiple strengthening, proprioceptive and plyometric exercises as rehabilitation progresses (Figure 6). The patient is cued to keep the knees behind the toes and to maintain the knees in neutral alignment in the frontal plane. Any hip adduction, internal rotation, or dynamic knee valgus is discouraged. Mirror feedback, a resistance band placed around the knees and other tactile cues are utilized to promote this movement pattern. The hip hike exercise is used to teach gluteal and pelvic control of a weight bearing position (Figure 7). The patient stands on the edge of a box and hikes the hip up on the non-stance limb while focusing on squeezing the gluteal muscles on the stance limb. Once proper squat form and the hip hike have been mastered, a single limb squat progression can begin (Figure 8). The single limb squat has demonstrated greater than $70 \%$ MVIC for gluteus maximus and medius 3 and requires good lower extremity and trunk control. This often starts in a small range with upper extremity support. The same cues given for the double limb squat are carried over to the single limb squat. The patient is to maintain good frontal plane alignment of the hip and knee, as well as not allowing the knee to travel anterior to the toes.

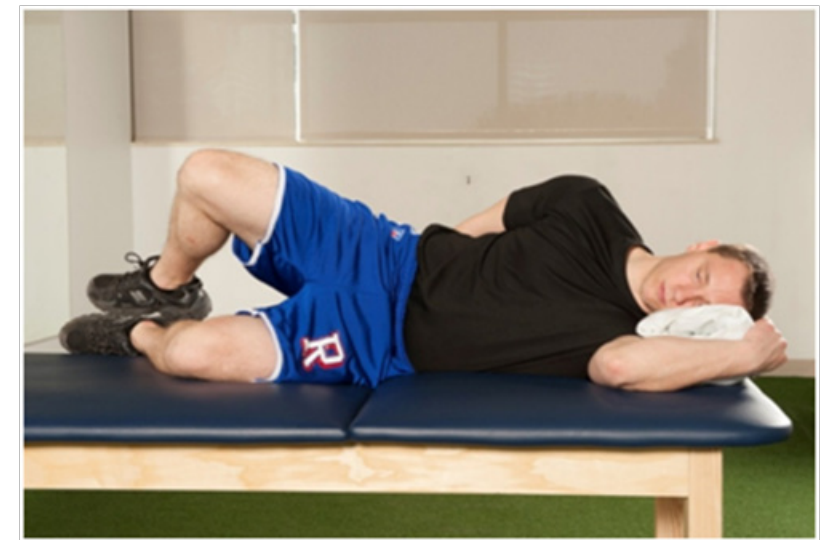

Figure 4 Clam Shell. The patient keeps the feet together and gently lifts the top knee up toward the ceiling, squeezing the gluteal muscles.

Although the quadriceps and hip control have been highlighted here, a plethora of other interventions are used by the therapist based on his/her ongoing assessment of strengths and weaknesses. Once the patient can perform 20 single limb squats with good hip and quadriceps control, a return to run program and a plyometric program are introduced. The return to run program begins very basically with running 1 minute and walking 5 minutes for 4 rounds. This program is progressed by $10 \%$ each week as long as the patient does not have any pain. Plyometrics begin with simple double limb jumps in place, 
focusing on proper mechanics early on rather than outright power. The plyometrics and return to run program are strategically progressed to increase the load on the healing structures to allow appropriate overload and adaptation of the tissues. Return to sport is based upon a report of no pain, no instability and full strength with manual muscle testing and excellent mechanics with single limb squat. In addition, single limb hop tests, consisting of single limb hop for distance, triple limb hop for distance, single limb hop for time and crossover hop for distance are performed by the patient. The limb symmetry index must be greater than or equal to $90 \%$ and hop mechanics must be excellent as qualitatively assessed by the physical therapist.
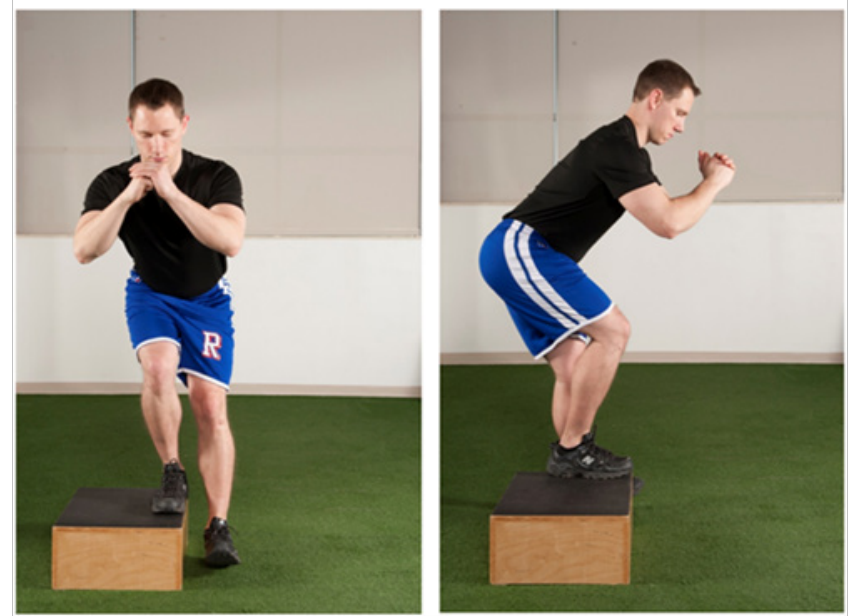

Figure 5 Side-lying Hip Abduction - The therapist ensures that the patient is not compensating with his/her hip flexors and that the hip is in slight extension and external rotation to ensure the gluteals are being targeted.

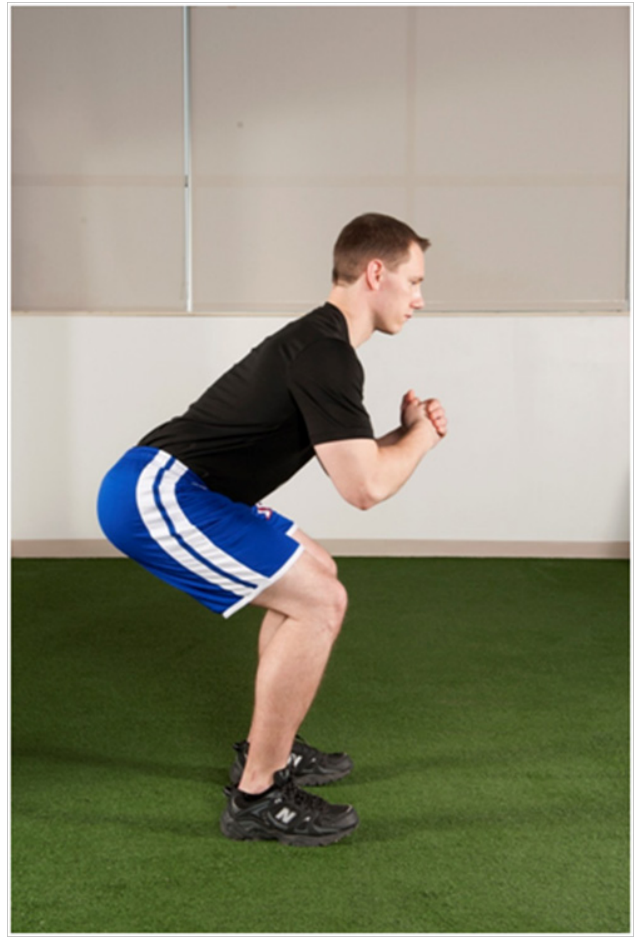

Figure 6 The Body Weight Squat. The patient is cued to keep the knees behind the toes and to maintain the knees in neutral alignment in the frontal plane. Any hip adduction, internal rotation, or dynamic knee valgus is discouraged. Mirror feedback, a resistance band theraband placed around the knees, and other tactile cues are utilized to promote this movement pattern.

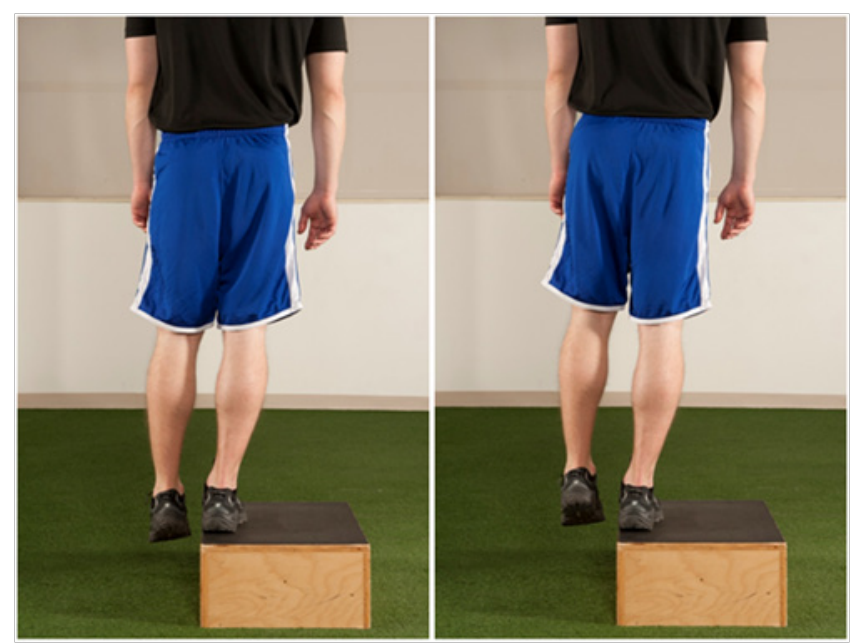

Figure 7 Hip hike. The patient stands on the edge of a box and hikes the hip up on the non-stance limb while focusing on squeezing the gluteal muscles on the stance limb.

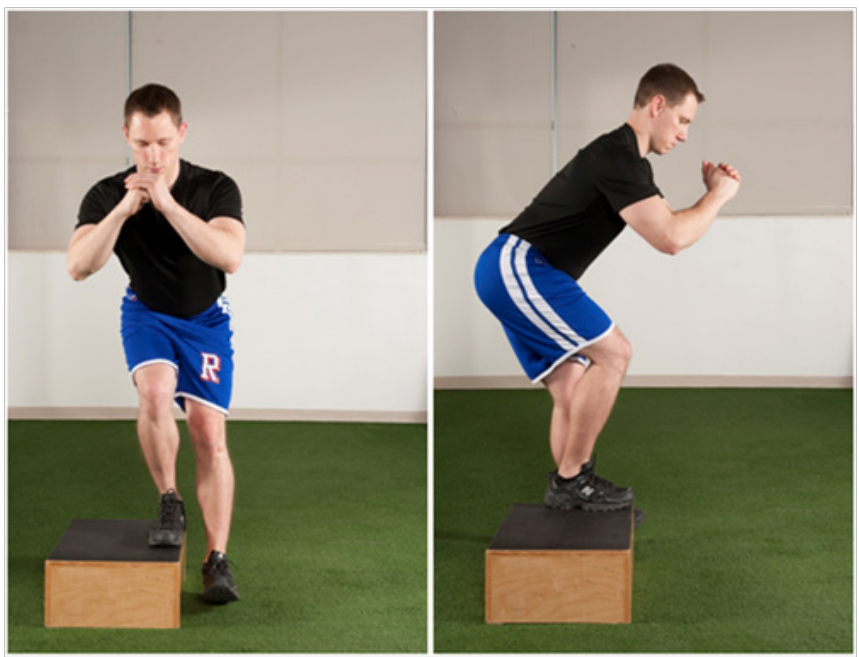

Figure 8 Single Limb Squat. The patient is cued to maintain good frontal plane alignment of the hip and knee, as well as not allowing the knee to travel anterior to the toes. This exercise often starts on a much smaller platform and is then elevated as patient progresses through rehabilitation.

\section{Statistical analysis}

The primary outcome measures were time until return to play and redislocation rates. These calculations were based on the patient's ability to return to his or her stated level of function prior to injury. One patient did not return for follow-up evaluation. This patient was contacted and asked about level of activity and whether he/she had returned to play and was then included in the data set. Patients were stratified based on mechanism of injury, sex and the sport played and compared with one another using $\mathrm{t}$ and $\mathrm{z}$ tests. The $\mathrm{t}$ tests were set to two tails, as there were no predicted outcomes in our population sets. Statistical significance was defined a priori as $\mathrm{P}<0.05$.

\section{Results}

Nineteen patients met inclusion criteria (mean age, 15.2years; range, $11-19$ years). Fifteen patients were male ( $79 \%$ of our data set) and four were female (21\%). The average time to return to previous level of activity was $7 \pm 2$ months (Figure 2) and there were three 
recurrent dislocations. There were no significant differences in return to sporting activities according to mechanism of injury $(\mathrm{P}=0.67$; Table $1 \&$ Figure 9$)$, sex $(\mathrm{P}=0.77$; Table 2$)$, or sporting activity $(\mathrm{P}=0.91$; Table 3 \& Figure 10). All preoperative MRI findings of intra-articular pathology were confirmed intra-operatively by arthroscopy. We contacted patients by phone to obtain a Kujala score following their primary MPFL repair. Of the 19 patients, 11 phone numbers were disconnected or changed, leaving eight patients with a mean score of 96.3 from their date of surgery (45-81 months).

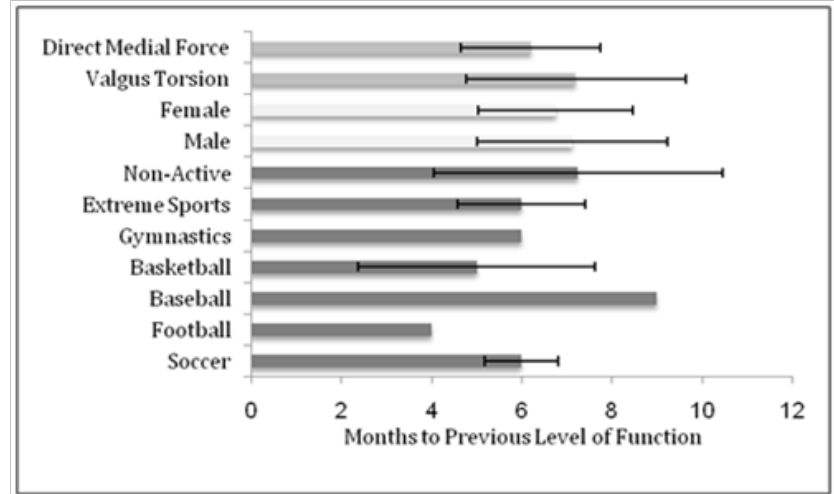

Figure 9 Summary of Clinical Data and Return to Previous Level of Function. Data did not vary significantly based on mechanism of injury, gender, or activity to be resumed.

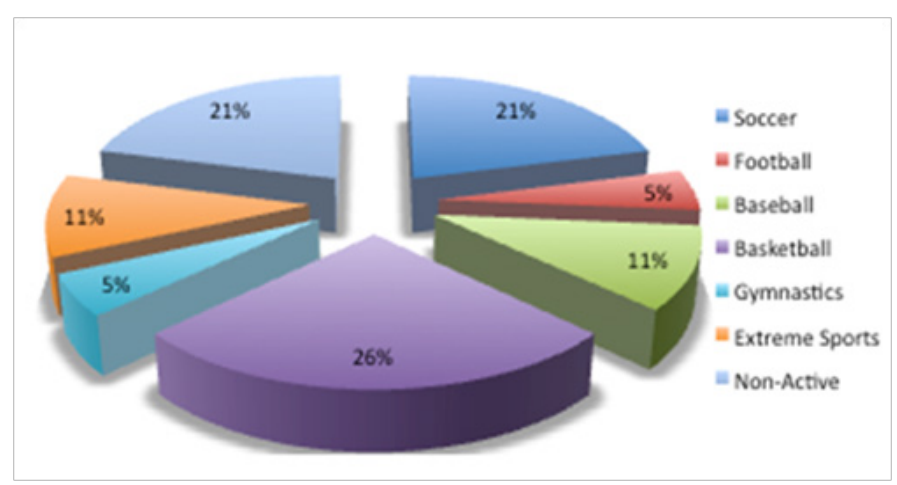

Figure 10 Summary of clinical data and return to previous level of function. Data did not vary significantly based on mechanism of injury, gender, or activity to be resumed.

Table I Data on the patients, stratified by mechanism of injury

\begin{tabular}{lllll}
\hline $\begin{array}{l}\text { Mechanism of } \\
\text { injury }\end{array}$ & $\begin{array}{l}\text { Number } \\
\text { of } \\
\text { patients }\end{array}$ & $\begin{array}{l}\text { Percentage } \\
\text { of data set }\end{array}$ & $\begin{array}{l}\text { Average } \\
\text { return to } \\
\text { play }\end{array}$ & $\begin{array}{l}\text { Standard } \\
\text { deviation value }\end{array}$ \\
\hline $\begin{array}{l}\text { Valgus Torsion on } \\
\text { a Planted Surface }\end{array}$ & $53 \%$ & 7.2 & 2.4 & 0.67 \\
$\begin{array}{l}\text { Direct Medial } \\
\text { Knee Trauma }\end{array}$ & 4 & $47 \%$ & 6.2 & 1.5 \\
\end{tabular}

Table 2 Data on the patients, stratified by sex

\begin{tabular}{lllll}
\hline Sex $\begin{array}{l}\text { Number } \\
\text { of } \\
\text { patients }\end{array}$ of data set & $\begin{array}{l}\text { Average } \\
\text { return to } \\
\text { play }\end{array}$ & $\begin{array}{l}\text { Standard } \\
\text { deviation }\end{array}$ \\
\hline Male value \\
\hline
\end{tabular}

\section{Discussion}

The usual mechanism of injury in MPFL tears is a twisting force applied to a flexed knee with the femur rotating internally while the foot is planted during athletic play. Injury more rarely occurs via a direct medial blow to the patella. In either situation, the varus forces applied to the patella must exceed the resisted biomechanical support and structure of the distal-lateral femoral trochlear groove, the MPFL, the patellomeniscal ligament and the medial retinaculum identified by Warren \& Marshall ${ }^{13}$. Concomitant with pediatric patellar dislocation there is often an MPFL tear or attenuation. Patients will often present with a spontaneously reduced patella; thus early management centers around the acute inflammatory response and diagnosing severe vastus medialis avulsions or osteochondral fractures. ${ }^{14}$

Table 3 Data on the patients, stratified by sport to which they returned

\begin{tabular}{lllll}
\hline Sport & $\begin{array}{l}\text { Number } \\
\text { of } \\
\text { patients }\end{array}$ & $\begin{array}{l}\text { Average } \\
\text { return } \\
\text { to play } \\
\text { (months) }\end{array}$ & $\begin{array}{l}\text { Standard } \\
\text { deviation }\end{array}$ & P value \\
\hline Soccer & 4 & 5.7 & 0.8 & 0.91 \\
Football & 1 & 4 & 0 & \\
Baseball & 2 & 9 & 0 & \\
Basketball & 5 & 5 & 2.6 \\
Gymnastics & 1 & 6 & 0 \\
Extreme & 2 & 6 & 1.4 \\
Sports & & & 3.201 \\
Others & 4 & 7.25 &
\end{tabular}

Several surgical options exist. In this study, MPFL tears with concomitant intra-articular pathology underwent an arthroscopic analysis of the soft and bony tissue of the knee joint along with an open MPFL repair. MPFL reconstruction is another option, but debate continues regarding the effectiveness of this procedure. ${ }^{15,16}$ A study by Servien E et al. ${ }^{17}$ illustrates the difficulty with reliably placing a femoral tunnel for MPFL reconstruction. They analyzed 29 knees 1 year postoperatively to assess the femoral tunnel placement radiographically and they found that 10 femoral tunnels were in a high and/or anterior and high position. Bollier $\mathrm{M}$ et al. ${ }^{18}$ showed that femoral tunnel malpositioning led to medial subluxation, medial 
patellofemoral articular overload and recurrent lateral instability. Elias JJ \& Cosgarea $\mathrm{AJ}^{19}$ qualified the effects of errors in graft length and tunnel position, by analyzing the subsequent forces and pressures across the medial patellofemoral cartilage. Their study linked tunnel malposition and incorrect graft length to increased pain, arthrosis, patellar fractures and cartilage degradation. Sillanpää PJ et al. ${ }^{20}$ reported that the MPFL tears from the femur in $66 \%$ of cases, from the patella in $13 \%$ of cases and from the mid substance in $21 \%$ of cases. Putney SA et al. ${ }^{21}$ studied 113 children aged 5 to 17 years and noted that $73 \%$ of MPFL injuries were at the adductor tubercle. Another recent study ${ }^{22}$ showed that 50 consecutive patients aged 10.5 to 17.5 years who were operated on for injuries after a first episode of traumatic patellar dislocation showed mid substance MPFL tears, demonstrating age-related MPFL injuries. Therefore, we prefer to avoid the possible complications of femoral tunnel placement by repairing the MPFL with an imbrication technique when the tear is found to be midsubstance.

Nonsurgical management is an option for MPFL injuries. Sillanpää $\mathrm{PJ}$ et al. ${ }^{20}$ retrospectively analyzed 53 patients treated nonoperatively for MPFL injuries confirmed by MRI findings. Patients were followed for 7 years; 15 patients reported patellar instability and nine had frank patellar dislocations. Control MRIs showed that $50 \%$ of patients had a full-thickness patellofemoral cartilage lesion. Hinton RY \& Sharma $\mathrm{KM}^{5}$ state that osteochondral lesions are a major contributor to adverse outcomes associated with MPFL tears. Given the high rate of patellar instability and suboptimal results with nonoperative management documented in the literature, we advocate an MPFL repair or reconstruction when an MPFL tear is noted in the setting of intra-articular pathology.

Tom A \& Fulkerson $\mathrm{JP}^{23}$ studied 13 consecutive cases in which an MPFL remnant was stretched, elongated and healed after a patellar dislocation. The MPFL was explored using a mini-open incision, noted intraoperatively to be attenuated and primarily repaired to restore the patellar stability. This study is consistent with our preoperative MRI and associated intraoperative findings, leading us to recommend repair of the midsubstance tear of the MPFL associated with intra-articular pathology. A primary repair has been shown to restore patellar stability in patients who have had dislocations and offers a viable alternative to a full MPFL reconstruction, without its attendant risks of autograft donor site morbidity or allograft infection or disease transmission.

Although a direct comparison cannot be made owing to the differences in chronicity of the dislocations, it is encouraging to compare our postoperative protocols with those reported by Arendt EA et al. ${ }^{24}$ who retrospectively reviewed clinical outcomes of MPFL repairs in chronic lateral patellar dislocations. In their study, 55 knees underwent MPFL repair; however, only 22 patients did not experience recurrent dislocations. The authors allowed patients partial weight bearing with $20^{\circ}$ of flexion in a hinged knee brace, in contrast to our protocol, which involves 4 weeks of non weight bearing followed by weight bearing as tolerated.

Careful perioperative management and follow-up are also necessary for optimal outcomes. After perioperative management and early healing, physical therapy is indicated to strengthen the lateral and medial stabilizers of the knee. ${ }^{25}$ The healthcare team should manage the patient's progress closely. This includes open communication between the orthopedist, the physical therapy team and the patient. Several investigators have identified a link between abnormal hip strength and patellofemoral pain. ${ }^{10-12,26,27}$ Excessive hip adduction and internal rotation can cause a decrease in the contact area between the patella and trochlea and a subsequent increase in the lateral joint stress. ${ }^{28-30}$ Earl JE \& Hoch $\mathrm{AZ}^{31}$ found that an 8-week rehabilitation program addressing neuromuscular control of the hip and core musculature reduced the knee abduction moment and significantly improved functional outcomes. Nakagawa TH et al. ${ }^{32}$ found that hip abductor and external rotator strengthening paired with quadriceps strengthening resulted in significantly greater reductions in pain with functional activity compared to a group receiving quadriceps strengthening alone. ${ }^{20}$ In addition to the regimented postoperative weight bearing, it is believed that patients undergoing MPFL repair will benefit from the rehabilitation approach described above, as the goal is to prevent excessive lateral translation of the patella and decrease stress on the healing articular cartilage and MPFL repair. ${ }^{31,33}$

In a systematic review of first-time acute traumatic dislocations, Stefancin JJ \& Parker RD ${ }^{34}$ identified recurrent dislocation, patellar instability, pain, decreased sporting activity and osteoarthritis of the patellar compartment as likely outcomes for these patients. These studies also recommend surgical intervention based on the criteria of osteochondral fracture, substantial disruption of the medial patellar stabilizers, a laterally subluxated patella with normal alignment of the contralateral knee, or a second dislocation, or in patients not improving with appropriate rehabilitation.

Strengths of our study include the uniformity of surgical criteria, allowing for a set protocol. A single surgeon using the same surgical technique completed all procedures. The protocol may not be useful, however, for surgeons who use other techniques for MPFL repair. The number of subjects limits the power of this study. A larger data set would increase the power; however, this is the largest study of solely pediatric patients, with an average age of 15 years and a maximum age of 19 years. According to our literature review through Pubmed and Medline, our study represents the longest Kujala score followup for a primary midsubstance MPFL repair, although 11 patients were lost to follow-up. Demographic and other risk factors for these injuries should be identified, in addition to those we analyzed.

Another limitation of our study is its retrospective design. A prospective study might help define the importance of surgical technique and postoperative management of midsubstance MPFL tears. Our study also lacked a direct control group; however, we have endeavored to compare our results with the current literature.

Our data suggest that with immediate employment of imaging, proper surgical repair, rehabilitation and early follow-up, original function can be restored in a timely manner for a specific injury pattern in MPFL injuries with chondral lesions.

\section{Acknowledgments}

None.

\section{Conflicts of interest}

The authors declare there is no conflict of interest.

\section{References}

1. Desio SM, Burks RT, Bachus KN. Soft tissue restraints to lateral patellar translation in the human knee. Am J Sports Med. 1998;26(1):59-65.

2. Larsen E, Lauridsen F. Conservative treatment of patellar dislocations. 
Influence of evident factors on the tendency to redislocation and the therapeutic result. Clin Orthop Relat Res. 1982;(171):131-136.

3. Sallay PI, Poggi J, Speer KP, et al. Acute dislocation of the patella. A correlative pathoanatomic study. Am J Sports Med. 1996;24(1):52-60.

4. Fisher B, Nyland J, Brand E, et al. Medial patellofemoral ligament reconstruction for recurrent patellar dislocation: a systematic review including rehabilitation and return-to-sports efficacy. Arthroscopy. 2010;26(10):1384-1394

5. Hinton RY, Sharma KM. Acute and recurrent patellar instability in the young athlete. Orthop Clin North Am. 2003;34(3):385-396.

6. Balcarek P, Walde TA, Frosch S, et al. MRI but not arthroscopy accurately diagnoses femoral MPFL injury in first-time patellar dislocations. Knee Surg Sports Traumatol Arthrosc. 2012;20(8):1575-1580.

7. Seeley M, Bowman KF, Walsh C, et al. Magnetic resonance imaging of acute patellar dislocation in children: patterns of injury and risk factors for recurrence. J Pediatr Orthop. 2012;32(2):145-155.

8. Powers CM, Ward SR, Fredericson M, et al. Patellofemoral kinematics during weight-bearing and non-weight-bearing knee extension in persons with lateral subluxation of the patella: a preliminary study. $J$ Orthop Sports Phys Ther. 2003;33(11):677-685

9. Palmieri-Smith RM, Thomas AC, Wojtys EM. Maximizing quadriceps strength after ACL reconstruction. Clin Sports Med. 2008;27(3):405424 .

10. Barton CJ, Lack S, Malliaras $\mathrm{P}$, et al. Gluteal muscle activity and patellofemoral pain syndrome: a systematic review. $\mathrm{Br} J$ Sports Med. 2013;47(4):207-214

11. Lankhorst NE, Bierma-Zeinstra SM, van Middelkoop M. Factors associated with patellofemoral pain syndrome: a systematic review. $\mathrm{Br} J$ Sports Med. 2013;47(4):193-206.

12. Moyano FR, Valenza MC, Martin LM, et al. Effectiveness of different exercises and stretching physiotherapy on pain and movement in patellofemoral pain syndrome: a randomized controlled trial. Clin Rehabil. 2013;27(5):409-417.

13. Palmu S, Kallio PE, Donell ST, et al. Acute patellar dislocation in children and adolescents: a randomized clinical trial. J Bone Joint Surg Am. 2008;90(3):463-470.

14. Warren LF, Marshall JL. The supporting structures and layers on the medial side of the knee: an anatomical analysis. J Bone Joint Surg Am. 1979;61(1):56-62.

15. Bedi H, Marzo J. The biomechanics of medial patellofemoral ligament repair followed by lateral retinacular release. Am J Sports Med. 2010;38(7):1462-1467.

16. LeGrand AB, Greis PE, Dobbs RE, et al. MPFL reconstruction. Sports Med Arthrosc. 2007;15(2):72-77.

17. Servien E, Fritsch B, Lustig S, et al. In vivo positioning analysis of medial patellofemoral ligament reconstruction. Am J Sports Med. 2011;39(1):134-139.

18. Bollier M, Fulkerson J, Cosgarea A, et al. Technical failure of medial patellofemoral ligament reconstruction. Arthroscopy. 2011;27(8):1153-
1159.

19. Elias JJ, Cosgarea AJ. Technical errors during medial patellofemoral ligament reconstruction could overload medial patellofemoral cartilage: a computational analysis. Am J Sports Med. 2006;34(9):1478-1485.

20. Sillanpaa PJ, Peltola E, Mattila VM, et al. Femoral avulsion of the medial patellofemoral ligament after primary traumatic patellar dislocation predicts subsequent instability in men: a mean 7-year nonoperative follow-up study. Am J Sports Med. 2009;37(8):1513-1521.

21. Putney SA, Smith CS, Neal KM. The location of medial patellofemoral ligament injury in adolescents and children. $J$ Pediatr Orthop. 2012;32(3):241-244.

22. Felus J, Kowalczyk B. Age-related differences in medial patellofemoral ligament injury patterns in traumatic patellar dislocation: case series of 50 surgically treated children and adolescents. Am J Sports Med. 2012;40(10):2357-2364

23. Tom A, Fulkerson JP. Restoration of native medial patellofemoral ligament support after patella dislocation. Sports Med Arthrosc. 2007;15(2):68-71

24. Arendt EA, Moeller A, Agel J. Clinical outcomes of medial patellofemoral ligament repair in recurrent (chronic) lateral patella dislocations. Knee Surg Sports Traumatol Arthrosc. 2011;19(11):1909-1914.

25. McConnell J. Rehabilitation and nonoperative treatment of patellar instability. Sports Med Arthrosc. 2007;15(2): 95-104.

26. Noehren B, Hamill J, Davis I. Prospective evidence for a hip etiology in patellofemoral pain. Med Sci Sports Exerc. 2013;45(6):1120-1124.

27. Rixe JA, Glick JE, Brady J, et al. A review of the management of patellofemoral pain syndrome. Phys Sports med. 2013;41(3):19-28.

28. Lee TQ, Anzel SH, Bennett KA, et al. The influence of fixed rotational deformities of the femur on the patellofemoral contact pressures in human cadaver knees. Clin Orthop Relat Res. 1994;(302):69-74.

29. Powers CM. The influence of abnormal hip mechanics on knee injury: a biomechanical perspective. J Orthop Sports Phys Ther. 2010;40(2):4251

30. Salsich GB, Perman WH. Patellofemoral joint contact area is influenced by tibiofemoral rotation alignment in individuals who have patellofemoral pain. J Orthop Sports Phys Ther. 2007;37(9):521-528.

31. Earl JE, Hoch AZ. A proximal strengthening program improves pain, function, and biomechanics in women with patellofemoral pain syndrome. Am J Sports Med. 2011;39(1):154-163.

32. Nakagawa TH, Muniz TB, Baldon Rde M, et al. The effect of additional strengthening of hip abductor and lateral rotator muscles in patellofemoral pain syndrome: a randomized controlled pilot study. Clin Rehabil. 2008;22(12):1051-1060.

33. Bicos J, Fulkerson JP, Amis A. Current concepts review: the medial patellofemoral ligament. Am J Sports Med. 2007;35(3):484-492.

34. Stefancin JJ, Parker RD. First-time traumatic patellar dislocation: a systematic review. Clin Orthop Relat Res. 2007;455:93-101. 